\title{
Mass of the Milky Way
}

\author{
O. I. Wong
}

School of Physics, University of Melbourne, VIC 3010, Australia

M. J. Drinkwater; J. B. Jones

Physics Department, University of Queensland, QLD 4072, Australia; Sch. of Physics \& Ast., Univ. of Nottingham, Nottingham NG72RD, UK

M. D. Gregg; K. C. Freeman

Department of Physics, University of California, Davis, CA 95616, USA; Res. School of Astronomy \& Astrophysics, MSO, ACT 2611, Australia

\begin{abstract}
We present a new estimate of the mass of the Milky Way based on the escape velocity of a sample of distant stars, about $12 \mathrm{kpc}$ from the Galactic centre and about $5 \mathrm{kpc}$ from the plane of the Galaxy. Our sample is very different from previous escape-velocity studies, being compiled from an all-object spectroscopic survey of a region of sky. The derived mass within $12 \mathrm{kpc}$ of the Galactic centre is $(1.3 \pm 0.3) \times 10^{11} \mathrm{M}_{\odot}$.
\end{abstract}

\section{Introduction and high-velocity star sample}

Previous applications of the escape velocity method have identified bright high velocity stars from proper motion studies and have therefore been limited to stars in the Solar Neighbourhood. Our study, based on radial velocities of 4800 randomly-selected stars, is not limited to nearby stars. Indeed they are mostly greater than $10 \mathrm{kpc}$ distant so we are probing the gravitational field of a new region of the Milky Way. Fig. 1(A) shows the results of previous determinations of the Galactic mass at various radii from the centre of the Galaxy.

The Fornax Cluster Spectroscopic Survey (FCSS; Drinkwater et al. 2000, $\mathrm{A} \& \mathrm{~A}, 355,900)$ is a complete, all-object redshift survey of optically-selected targets within a $b_{j}=16.5$ to 19.7 magnitude range in the direction of the Fornax Cluster of galaxies. The survey is being conducted with the $2 \mathrm{dF}$ multi fibre spectrograph on the Anglo-Australian Telescope (AAT) at 3600-8010 Fig. 1(B) shows the velocity-colour distribution for the entire set of 4800 stars identified with $2 \mathrm{dF}$. The graph shows distinct blue and red populations (see also the discussion in Drinkwater et al. 2000). It can be seen that the blue population has a broader distribution of velocities than the red population, with a tail to large positive velocities, the subject of this study. 

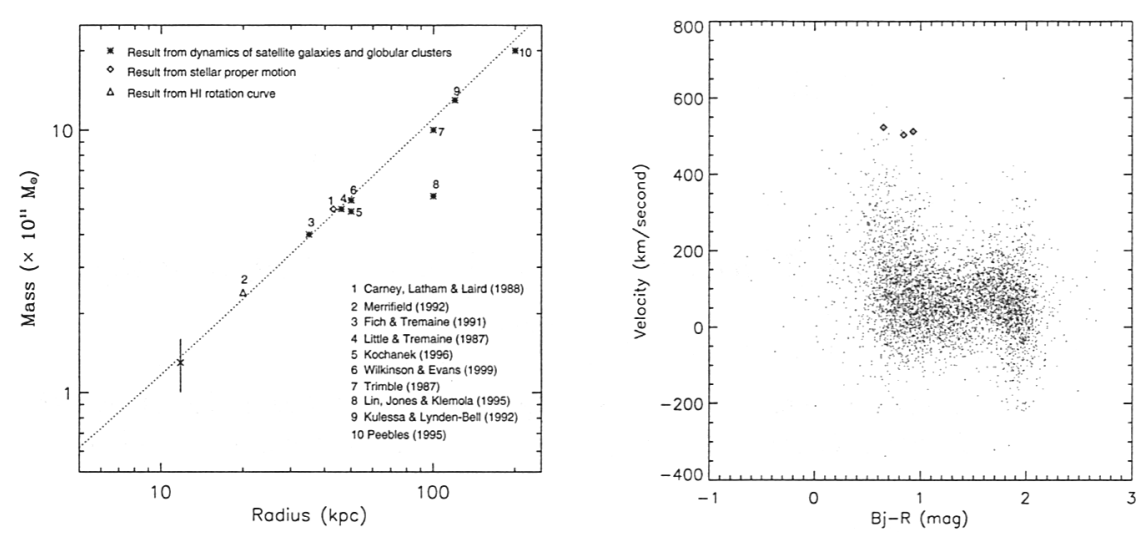

Figure 1. (A) Left: Estimates of the Galactic mass plotted as a function of radius from the Galactic centre. Results from previous studies are plotted with asterisks, triangles \& diamond-shaped points and the results from this project are plotted with a cross. The dotted straight line shows the linear relation between the mass and the radius of the Galaxy. (B) Right: colour-velocity plot of 4800 stars found in the FCSS. The blue star population has a higher average velocity than the red stars. The diamond points represent the 3 high-velocity stars observed at higher resolution.

\section{Analysis and conclusions}

The heliocentric velocity range of the sample stars is -431 to $577 \mathrm{~km} \mathrm{~s}^{-1}$ but high-resolution spectra showed that the highest values were over-estimates, 2sigma extremes of the error distribution in the $2 \mathrm{dF}$ data $\left(\sigma_{v} \approx 70 \mathrm{~km} \mathrm{~s}^{-1}\right)$. We used Monte Carlo simulations of the true velocity distribution smeared by Gaussian random errors to estimate the true escape velocity of our star sample. A maximum Galactocentric radial velocity of $312 \pm 20 \mathrm{~km} \mathrm{~s}^{-1}$ was obtained from these simulations.

To estimate the distance of our sample we note that with a standard multicomponent model of the Galaxy, the high-velocity objects will be halo stars rather than disk stars (Fig. 1(B)). The distances were estimated from photometric parallaxes determined by the method used by Gilmore, Wyse \& Jones (1995, AJ, 109, 1095), based on the calibration of Laird, Carney \& Latham (1988, AJ, 95, 1843). The mean distance of the high-velocity stars from the Sun was found to be $6.31 \pm 2.1 \mathrm{kpc}$. This corresponds to a mean distance of these stars from the centre of the Galaxy of $11.8 \pm 2.1 \mathrm{kpc}$.

Assuming that the lower limit of the escape velocity is $312 \pm 20 \mathrm{~km} \mathrm{~s}^{-1}$ and the radius from the Galactic centre to these fast stars is $11.8 \pm 2.1 \mathrm{kpc}$, the lower bound to the mass of the Milky Way is $(1.3 \pm 0.3) \times 10^{11} M_{\odot}$. Taking the luminosity of the Milky Way to be $2.3 \times 10^{10} L_{\odot}$, the mass to light ratio of the Milky Way at a radius of $11.8 \mathrm{kpc}$ is $5.7 M_{\odot} / L_{\odot}$. 